\title{
Decompression only versus fusion in octogenarians with spinal epidural abscesses: early complications, clinical and radiological outcome with two years follow-up
}

\section{Pavlina Lenga ( $\sim$ pavlina.lenga@med.uni-heidelberg.de)}

University Hospital Heidelberg: UniversitatsKlinikum Heidelberg https://orcid.org/0000-0003-3842-0783

Gelo Gülec

University Hospital Heidelberg: UniversitatsKlinikum Heidelberg

\section{Awais Akbar Bajwa}

University Hospital Heidelberg: UniversitatsKlinikum Heidelberg

\section{Mohammed Issa}

vUniversity Hospital Heidelberg: UniversitatsKlinikum Heidelberg

\section{Rod Oskouian}

Swedish Neuroscience Institute

Jens Chapman

Swedish Neuroscience Institute

\section{Karl Kiening}

University Hospital Heidelberg: UniversitatsKlinikum Heidelberg

\section{Andreas Unterberg}

University Hospital Heidelberg: UniversitatsKlinikum Heidelberg

\section{Basem Ishak}

University Hospital Heidelberg: UniversitatsKlinikum Heidelberg

\section{Research Article}

Keywords: surgical decompression, instrumentation, octogenarians, epidural abscess, osteomyelitis

Posted Date: March 2nd, 2022

DOl: https://doi.org/10.21203/rs.3.rs-1389748/v1

License: () (1) This work is licensed under a Creative Commons Attribution 4.0 International License. Read Full License 


\section{Abstract}

Despite increased life expectancy due to healthcare quality improvements globally, pyogenic vertebral osteomyelitis (PVO) treatment with a spinal epidural abscess (SEA) remains challenging in patients older than 80 years. We aimed to assess octogenarians for PVO prevalence with SEA and compare after-surgery clinical outcomes of decompression and decompression and instrumentation. A retrospective review of electronic medical records at a single institution was conducted between September 2005 and December 2020. Patient demographics, surgical characteristics, complications, hospital course, and 90-day mortality were collected. Comorbidities were assessed using the age-adjusted Charlson comorbidity index (CCI). Over 16 years, 35 patients aged $\geq 80$ years with PVO and SEA were identified. Eighteen patients underwent surgical decompression ("decompression group"), and 17 underwent surgical decompression with instrumentation ("instrumentation group"). Both groups had a $\mathrm{CCl}>6$ (mean $\pm \mathrm{SD}, 8.9 \pm 2.1$ vs. $9.6 \pm 2.7$, respectively; $p=$ 0.065 ). Instrumentation group patients had a significantly longer hospital stay but no ICU stay. In-hospital and 90-days mortality rates were similar in both groups. The mean follow-up was $26.6 \pm 12.4$ months. No further surgeries were performed. Infection levels and neurological status were improved in both groups at discharge. At the second-stage analysis, significant improvements in the blood infection parameters and the neurological status were detected in the decompression group. Octogenarians with PVO and SEA have a high adverse events risk after surgical procedures. Surgical decompression might contribute to earlier clinical recovery in older patients. Thus, the surgical approach should be discussed with patients and their relatives and be carefully weighed.

\section{Introduction}

Despite accelerating improvements in the quality of health care worldwide, pyogenic vertebral osteomyelitis (PVO) with concomitant spinal epidural abscess (SEA) remains a potentially devastating condition. PVO is caused by bacterial infection of the vertebral bodies that can extend into the adjacent intervertebral disc [5], while SEA refers to a collection of purulent material between the dura and the osseous-ligament structures of the spinal cord. In previous decades, the incidence of SEA was very low, ranging from 0.2 to 1.2 cases per 10,000 admissions $[3,7,13,20,21]$. However, the numbers are currently rising, with recent estimates ranging from 1.2 to 12.5 per 10,000 admissions $[2,6]$. This rapid increase is most likely attributable to the spread of intravenous (IV) drug abuse, an ever-aging population, an increase in spinal surgeries, rising comorbidities such as diabetes mellitus, and the presence of implants providing chronic vascular access $[7,17,19]$. While advanced imaging techniques, efficient IV antibiotics, and advances in surgical techniques have reduced mortality rates, the morbidity remains disturbingly high at $33-47 \%[7,17,19]$. Since both symptomatic vertebral osteomyelitis and SEA with neurological deficits are considered surgical emergencies, the main goal of treatment is expedient surgical decompression and antibiotic therapy. Importantly, studies clearly show that the time between the occurrence of neurological symptoms and surgical treatment is pivotal and predictive of neurological recovery $[7,15]$.

In the case of older and frail patients, the choice of therapy remains controversial, considering perioperative risks associated with patients' poor general clinical condition. The scarcity of robust evidence concerning the optimal treatment for PVO with SEA in older adults, especially in octogenarians, hampers physicians who must quickly decide whether to perform a surgical procedure. 
Owing to the lack of clinical evidence on this topic, we aimed to assess and compare the clinical course and determine morbidity and mortality rates after surgical decompression only versus surgical decompression with instrumentation, exclusively in octogenarians.

\section{Methods}

\section{Study design and patient characteristics}

We retrospectively evaluated the clinical and imaging data collected from our institution's database between September 2005 and December 2020. This study was approved by the local ethics committee of our institution (no. 880/2021) and conducted in accordance with the Declaration of Helsinki. The requirement for informed consent was waived because of the retrospective nature of the study. Patients aged $\geq 80$ years with PVO and SEA across the thoracic and lumbar spine were consecutively enrolled. The diagnosis was based on magnetic resonance imaging (MRI). Spine stability was examined by findings of computed tomography (CT). Exclusion criteria were: $<80$ years old, had a concurrent intracranial or cervical pathology, spinal instability and progressive kyphotic deformity, or the requisite data were unavailable. The electronic medical records were assessed to obtain patient demographics, comorbidities, American Society of Anesthesiologists (ASA) scores, duration of surgery, number of treated spinal levels, peri- and postoperative complications, hospital length of stay (LOS), intensive care unit (ICU) stay, readmissions, reoperations, and mortality. Comorbidities present before surgery were assessed using the age-adjusted Charlson comorbidity index (CCI) $[9,12]$. The $\mathrm{CCl}$ was calculated for each patient and classified as: no comorbidity $(\mathrm{CCl}=0)$, minimal comorbidity $(\mathrm{CCl}=1$ or 2$)$, moderate comorbidity $(\mathrm{CCl}=3-5)$, or severe comorbidity $(\mathrm{CCl}>5)$. The pre-treatment neurological condition was assessed using the motor score (MS) of the American Spinal Injury Association (ASIA) impairment grading system ( $M S=0$, no muscle strength; $M S=100$, healthy). The post-treatment MS was obtained from the last clinical encounter documented.

Routine clinical and radiological follow-up examinations were performed before discharge and at 3 months after surgery. The final follow-up varied between 3 and 72 months postoperatively. Standard radiographs in the anteroposterior and lateral views were obtained to evaluate screw position and fusion rate.

\section{Procedures}

Patients were allocated into two groups: 1) surgical decompression only (decompression group) and 2) surgical decompression with spinal instrumentation (instrumentation group). Decision-making was guided by the presenting neurological status (MS), the concomitant underlying pathologies, the extent of the pathology, and the discretion of an experienced treatment team consisting of neurosurgeons, neuroradiologists, and anesthesiologists. The final decision for decompression only or decompression with instrumentation was conducted by the surgeons' preference. A CT-based point-to-point navigation system was used to perform spinal instrumentation, as previously described by our study group [14].

In line with our institutional treatment protocols, blood samples or intraoperative cultures were collected prior to administration of IV antibiotics. Thereafter, IV antibiotics were initiated immediately. After identifying the bacterial specimens, the choice of IV antibiotics was adapted to reflect the antibiogram results. 


\section{Statistical analysis}

Categorical variables are presented as numbers and percentages. Continuous variables are presented as means \pm standard deviations and were verified as normally distributed using the Shapiro-Wilk test. Baseline characteristics, duration of surgery, number of treated spinal levels, perioperative and postoperative complications, LOS, ICU stay, readmissions, reoperations, and mortality were compared groupwise using independent $t$-tests for continuous variables and chi-squared tests for categorical variables. The Wilcoxon rank test was applied to evaluate changes in C-reactive protein (CRP), leukocytes, and neurological status (as MS) of each group at discharge. Because these procedures are uncommonly applied in patients aged $>80$ years, and because our sample was relatively small, we could not perform a multivariate analysis to adjust for potential confounders. A p-value $\leq 0.05$ was set as statistically significant.

\section{Results}

\section{Patient demographics and baseline characteristics}

Over a period of 15 years, 35 patients $\geq 80$ years of age diagnosed with PVO and SEA were enrolled in the study. Our sample had an overall mean age of $82.2 \pm 1.3$ years, with a predominance of men $(n=24,68.6 \%)$. Eighteen patients were allocated to the decompression group and 17 to the instrumentation group, respectively. No significant intergroup differences in comorbidities were observed. Both groups had a $\mathrm{CCl}>6$, indicating a poor prior baseline reserve $(8.9 \pm 2.1$ vs. $9.6 \pm 2.7$, respectively; $p=0.065)$. No significant differences were observed between the groups regarding the extent or location of the SEA. The lumbar spine was the most amenable region in both groups, followed by the thoracic region. Patients in both groups presented with a lumbar abscess with an extension to at least two levels. Blood infection parameters were relatively high, with CRP levels $173 \pm 15.8 \mathrm{mg} / \mathrm{L}$ in the surgical decompression group and $150.3 \pm 14.6 \mathrm{mg} / \mathrm{L}$ in the instrumentation group $(p<0.005)$. No differences were observed in motor impairment as measured by ASIA scoring (Table 1). 
Table 1

Baseline patient characteristics

\begin{tabular}{|c|c|c|c|}
\hline & $\begin{array}{l}\text { Decompression } \\
\mathrm{N}^{\mathrm{a}}=18\end{array}$ & $\begin{array}{l}\text { Instrumentation } \\
\mathrm{N}=17\end{array}$ & p-Value \\
\hline Age, y (mean, SD $\left.{ }^{b}\right)$ & $82.8(1.2)$ & $81.6(1.6)$ & 0.195 \\
\hline $\operatorname{Sex}\left(n^{c}, \%\right)$ & & & 0.328 \\
\hline Male & $11(61.1)$ & $13(76.5)$ & \\
\hline Female & $7(38.9)$ & $4(23.5)$ & \\
\hline $\mathrm{BMI}^{\mathrm{d}}, \mathrm{kg} / \mathrm{m}^{2}$ (mean, SD) & $27.4(5.0)$ & $26.8(3.7)$ & 0.610 \\
\hline \multicolumn{4}{|l|}{ Comorbidities } \\
\hline Age-adjusted $\mathrm{CCl}^{\mathrm{e}}$ score (mean, SD) & $8.9(2.1)$ & $9.6(2.7)$ & 0.065 \\
\hline Arterial hypertension (n, \%) & $14(77.8)$ & $13(76.5)$ & 0.927 \\
\hline Myocardial infarction (n, \%) & $1(5.6)$ & $5(29.4)$ & 0.061 \\
\hline Coronary heart disease (n, \%) & $12(66.7)$ & $9(52.9)$ & 0.407 \\
\hline Atrial fibrillation $(\mathrm{n}, \%)$ & $4(22.2)$ & $7(41.2)$ & 0.227 \\
\hline Heart failure (n, \%) & $6(33.3)$ & $5(29.4)$ & 0.803 \\
\hline $\operatorname{COPD}^{f}(\mathrm{n}, \%)$ & $3(16.7)$ & $7(41.2)$ & 0.109 \\
\hline Diabetes mellitus Type II (n, \%) & $5(29.4)$ & $8(47.1)$ & 0.290 \\
\hline Renal failure $(\mathrm{n}, \%)$ & $9(50.0)$ & $9(52.9)$ & 0.862 \\
\hline Liver disease (n, \%) & $2(11.1)$ & $3(17.6)$ & 0.339 \\
\hline Gastrointestinal ulcer (n, \%) & $3(16.7)$ & $4(23.5)$ & 0.618 \\
\hline TIA $\%$ stroke $(n, \%)$ & $0(0.0)$ & $2(11.8)$ & 0.122 \\
\hline Malignancy (n, \%) & $2(11.1)$ & $3(17.6)$ & 0.530 \\
\hline Dementia (n, \%) & $1(5.6)$ & $1(5.9)$ & 0.932 \\
\hline Previous spinal surgery $(n, \%)$ & $4(22.2)$ & $3(17.6)$ & 0.671 \\
\hline $\operatorname{ASA}^{\mathrm{h}}$ class $(\mathrm{n}, \%)$ & & & 0.737 \\
\hline II & $3(16.7)$ & $2(11.8)$ & \\
\hline
\end{tabular}

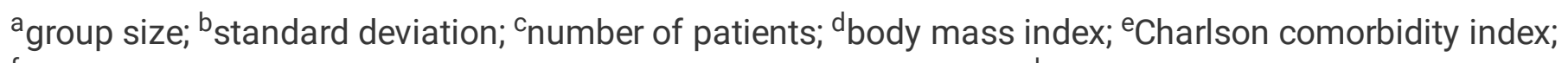
${ }^{f}$ chronic obstructive pulmonary disease; ${ }^{9}$ transient ischemic attack; ${ }^{\text {h}}$ American Society of Anesthesiologists; ${ }^{\mathrm{C}}$-reactive protein; ${ }^{\mathrm{j}}$ motor score of the American Spinal Injury Association grading system 


\begin{tabular}{|c|c|c|c|}
\hline & $\begin{array}{l}\text { Decompression } \\
\mathrm{N}^{\mathrm{a}}=18\end{array}$ & $\begin{array}{l}\text { Instrumentation } \\
\mathrm{N}=17\end{array}$ & p-Value \\
\hline III & $12(66.7)$ & $10(58.8)$ & \\
\hline IV & $3(16.7)$ & $3(17.6)$ & \\
\hline V & $0(0.0)$ & $1(5.9)$ & \\
\hline Localization of epidural abscess, $(n, \%)$ & & & 0.870 \\
\hline Thoracic & $8(44.4)$ & $8(47.1)$ & \\
\hline Thoracolumbar & $2(11.1)$ & $1(5.9)$ & \\
\hline Lumbar & $6(33.3)$ & $7(41.2)$ & \\
\hline Lumbosacral & $2(11.1)$ & $1(5.9)$ & \\
\hline CRP' level, mg/L (mean, SD) & $173.4(15.8)$ & $150.3(14.6)$ & 0.683 \\
\hline Leukocytes, count/L (mean, SD) & $11.9(5.6)$ & $12.2(4.8)$ & 0.946 \\
\hline Preoperative neurological deficit $(n, \%)$ & $16(88.9)$ & $15(88.2)$ & 0.330 \\
\hline Preoperative MS'j score (mean, SD) & $79.4(17.1)$ & $77.9(25.9)$ & 0.386 \\
\hline \multicolumn{4}{|c|}{$\begin{array}{l}\text { a group size; }{ }^{b} \text { standard deviation; }{ }^{c} \text { number of patients; }{ }^{d} \text { body mass index; }{ }^{e} \text { Charlson comorbidity index; } \\
\text { f chronic obstructive pulmonary disease; }{ }^{g} \text { transient ischemic attack; }{ }^{h} \text { American Society of } \\
\text { Anesthesiologists; }{ }^{i} \text { C-reactive protein; }{ }^{j} \text { motor score of the American Spinal Injury Association grading } \\
\text { system }\end{array}$} \\
\hline
\end{tabular}

\section{Surgical characteristics and clinical scores}

As shown in Table 2, the surgical duration of patients in the instrumentation group was significantly longer than that of those in the decompression-only group $(203.5 \pm 104.1 \mathrm{~min}$ vs. $132.8 \pm 89.7 \mathrm{~min} ; \mathrm{p}=0.007)$. In addition, the number of operated levels was significantly greater in patients with instrumentation than in patients with mere surgical decompression ( $3.7 \pm 1.6$ levels vs. $1.9 \pm 1.2$ levels; $p<0.001)$. Notably, while patients of the instrumentation group had significantly longer hospital LOS, the ICU stay was similar between both groups. Concerning the in-hospital mortality, no significant differences were obtained. The 90-day mortality rate was $23.5 \%$ in patients undergoing instrumentation versus $5.6 \%$ in patients with surgical decompression only; however, this difference did not reach statistical significance $(p>0.05)$. Moreover, in both groups, infection levels and neurological status showed significant improvement at discharge. Overall, the mean follow-up period was $26.6 \pm 12.4$ months, and no additional surgery was necessary due to secondary instability. Furthermore, no screw loosening or displacement was seen on radiographs. 
Table 2

Comparison of surgical characteristics and clinical course between groups

\begin{tabular}{|c|c|c|c|}
\hline & $\begin{array}{l}\text { Decompression } \\
\mathrm{N}^{\mathrm{a}}=18\end{array}$ & $\begin{array}{l}\text { Instrumentation } \\
\mathrm{N}=17\end{array}$ & p-value \\
\hline Surgical duration, min & $132.8(89.7)$ & $203.5(104.1)$ & $0.007 *$ \\
\hline No. of levels decompressed/fused & $1.9(1.2)$ & $3.7(1.6)$ & $<0.001^{*}$ \\
\hline Hospital stay, days & $8.9(6.2)$ & $14.8(10.4)$ & $0.020^{*}$ \\
\hline ICU ${ }^{b}$ stay, days & $3.1(2.3)$ & $4.2(3.1)$ & 0.405 \\
\hline \multicolumn{4}{|l|}{ Mortality } \\
\hline In-hospital (nc, \%) & $1(5.6)$ & $2(11.8)$ & 0.512 \\
\hline 90-day (n, \%) & $1(5.6)$ & $4(23.5)$ & 0.129 \\
\hline Post CRPd & $108.7(93.6)$ & $116.6(78.9)$ & 0.357 \\
\hline Delta CRP & $-64.7(68.2)$ & $-33.7(59.7)$ & 0.193 \\
\hline Post leukocytes & $10.0(5.1)$ & $10.6(3.5)$ & 0.760 \\
\hline Delta leukocytes & $-1.9(2.5)$ & $-1.6(5.4)$ & 0.858 \\
\hline Post MS & $83.6(15.5)$ & $80.0(24.2)$ & 0.935 \\
\hline Delta MS & $4.2(8.4)$ & $2.1(1.5)$ & 0.067 \\
\hline \multicolumn{4}{|c|}{ Except where otherwise indicated, quantities are mean (SD). } \\
\hline \multicolumn{4}{|c|}{ *significant difference; Post, after surgery; Delta, difference between pre-and post-surgical values. } \\
\hline
\end{tabular}

\section{Complications}

We observed a significant intergroup difference only in the occurrence of pleural effusion, with higher rates in the instrumentation group $(23.5 \%$ vs. $0.0 \%$; $p=0.029)$. A detailed breakdown of all recorded complications is provided in Table 3. Staphylococcus aureus was detected in about half of the patients in the instrumentation (70.6\%) and decompression (44.4\%) groups, either in blood or intraoperative samples. The next most frequent pathogen in both groups was Escherichia coli (instrumentation: 16.7\% vs. decompression groups: 11.8\%), followed by Enterococcus sp. (instrumentation: 11.1\% vs. decompression groups: 5.9\%), Pseudomonas aeruginosa (instrumentation: $5.6 \%$ vs. decompression groups: $5.6 \%$ ), and multiple pathogens (instrumentation: $5.6 \%$ vs. decompression groups: $5.6 \%$ ) respectively. No pathogens were identified in $16.7 \%$ of the decompression group and $5.9 \%$ of the decompression group. 
Table 3

Occurrence of adverse events

\begin{tabular}{|llll|}
\hline & $\begin{array}{l}\text { Decompression } \\
\mathbf{N}^{\mathrm{a}}=\mathbf{1 8}\end{array}$ & $\begin{array}{l}\text { Instrumentation } \\
\mathbf{N}=17\end{array}$ & p-value \\
\hline Deep wound infection & $1(5.6)$ & $2(11.8)$ & 0.512 \\
\hline Acute respiratory failure & $1(5.6)$ & $1(5.9)$ & 0.219 \\
\hline Acute heart failure & $1(5.6)$ & $2(11.8)$ & 0.296 \\
\hline Acute renal failure & $1(5.6)$ & $0(0.0)$ & 0.324 \\
\hline Septic shock & $0(0.0)$ & $2(11.8)$ & 0.967 \\
\hline Pneumonia & $2(11.1)$ & $5(29.4)$ & 0.228 \\
\hline Pleural effusion & $0(0.0)$ & $4(23.5)$ & $0.029 *$ \\
\hline Ileus & $0(0.0)$ & $1(5.9)$ & 0.296 \\
\hline Urinary tract infection & $0(0.0)$ & $1(5.9)$ & 0.296 \\
\hline All data are number of patients $(\%)$. & & \\
\hline *significant difference & & & \\
\hline a group size & & & \\
\hline
\end{tabular}

\section{Comparison of outcomes}

In a second-stage analysis, we examined the clinical outcomes in relation to the treatment strategies. After both types of surgery, a significant improvement was observed in blood infection parameters and neurological status at discharge compared to baseline measurements solely in the decompression group. In contrast, in the instrumentation group, statistical significance was reached only concerning the CPR levels. The clinical outcomes of both groups are provided in Table 4. 
Table 4

Comparison between baseline (before surgery) and discharge

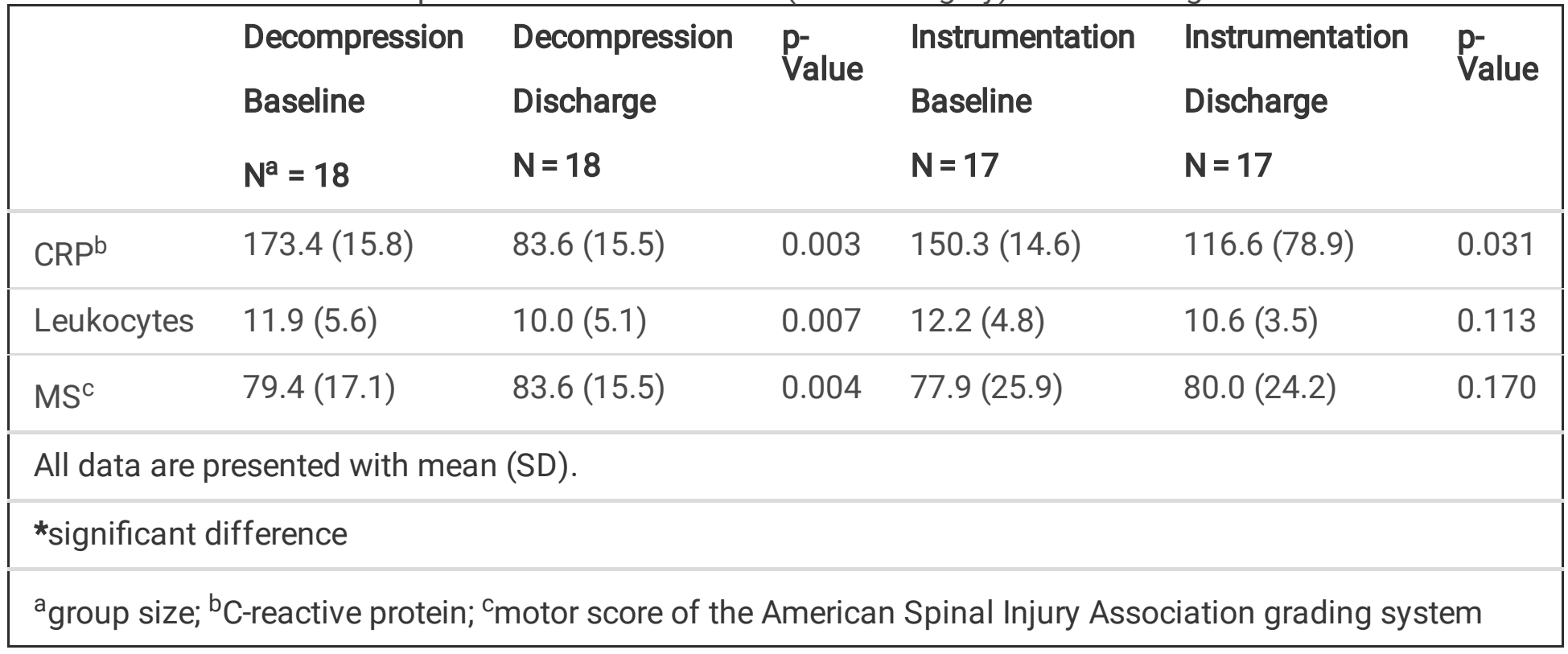

\section{Discussion}

Spinal epidural abscess is well known to be a devastating condition and is associated with high rates of neurological disability, ranging from $15-27 \%$, and mortality, ranging from $5-16 \%[7,8,20]$. Surgical procedures such as decompression are of paramount importance for obtaining a good clinical outcome and a satisfactory recovery. However, robust evidence comparing surgical strategies such as decompression only versus decompression with instrumentation in older patients remains scarce, especially in those aged $\geq 80$ years, who are exceptional because of their poor baseline reserve.

\section{Summary of findings}

To our knowledge, this is the first systematic analysis describing surgical strategies in octogenarians with PVO and concomitant SEA. The current study examined the clinical vignette, the neurological condition, the surgical characteristics, and the clinical course of this scarce disease with the aim of assessing morbidity and mortality rates. Interestingly, we found no significant differences between patients undergoing decompression only and those undergoing decompression with instrumentation regarding the following parameters: comorbidities, infection levels as determined by laboratory parameters (CRP and leukocytes), and grade of disability as defined by the MS score. Notably, patients undergoing pedicle screw fixation and surgical decompression had substantially longer surgical time and duration of hospitalization but no longer ICU stays. Also of note, among patients who opted for a surgical decompression procedure, we observed a significant improvement in the infection parameters and motor function at discharge, while in the instrumentation group, only the reduction in CRP levels reached statistical significance.

\section{Literature review}

Kim et al. retrospectively studied 16 patients aged $>65$ years with PVO and SEA. Almost all patients (87.5\%) underwent surgical decompression only [15]. Three patients were older than 80 years. Conversely, in the 
present study, we examined 35 patients older than 80 years, of whom 18 underwent surgical decompression only, and 17 underwent surgical decompression with instrumentation. Compared to Kim et al., who found a relatively low morbidity rate and a $\mathrm{CCl}$ as high as 4 in only 3 patients, our study cohort in both groups presented severe comorbidities with a mean $\mathrm{CCl}$ of 9.0 or greater [15]. Kim and colleagues did not report surgical duration, ICU stay, or hospital stay and observed a higher mortality rate after surgery (31.3\%) [15]. In contrast, the in-hospital mortality in our group undergoing surgical decompression only was fundamentally lower (5.6\%). The substantial discrepancy between the mortality rates might be attributable to the presence of delayed surgeries in the cited study, which were performed 40 days after the diagnosis. After completing the diagnostic work-up, our patients were operated on in less than $24 \mathrm{~h}$ with concurrent administration of IV antibiotics which might explain our low mortality rates even in older patients with multiple comorbidities.

Another retrospective analysis of 135 patients with PVO and SEA aged 18-88 years found that surgery led to significant improvements in neurological condition compared to medication alone [22]. It is important to highlight that about half of the cases were converted to surgical decompression and the other half to surgical decompression with instrumentation. However, the authors did not distinguish the outcomes of the two surgical approaches; thus, they broadly claimed that surgical treatment might be a critical pillar when treating patients with infections [22]. In another analysis of 60 patients with cervical SEAs and a mean age of 53 years, Alton et al. concluded that early surgery might be the key to the concurrent improvement of the infection and neurological statuses. Surprisingly, comorbidities did not predict treatment failure [1]. In agreement with these findings, Patel et al. compared medication versus surgical therapy in patients with PVO and SEA and advocated for early surgery based on an improvement of the MS by at least 3.4 points, also finding that diabetes mellitus, elevated CRP, and leukocytes were significant predictors of medical failure [18]. Based on the previous studies, the first-line therapy for such a devastating illness at our institution was surgery and not conservative management.

In a retrospective analysis of 40 patients with spinal abscess, Du et al. found that greater age ( $>60$ years) and the presence of comorbidities such as diabetes mellitus, respiratory, renal, or tumor diseases, and thrombocytopenia might significantly contribute to higher mortality rates evaluated 30 days post-surgery. They did not determine the surgical procedure [10]. Their overall 30-day mortality was $3.7 \%$, comparable to those found by Darouiche et al. and Vakili et al. [7, 23]. Interestingly, the most frequently reported complications were septic shock, cardiac arrest, and pneumonia which were associated with mortality. In contrast, our mortality rates were substantially higher at discharge (8.7\%) and at 90 days post-surgery (14.3\%). This phenomenon might be attributable to our patient subset consisting only of patients older than 80 years and having many compounding factors $(\mathrm{Cl}>6)$. Although each patient was postoperatively admitted to the ICU to prevent or treat postoperative complications, death was inevitable in some cases. Surprisingly, we found that older patients undergoing surgical decompression with instrumentation do not have a significantly greater mortality risk than surgical decompression alone. One explanation might be that the consistent use of spinal navigation in our surgical routine might be key for decreasing or even avoiding intraoperative complications and prolonged surgeries, thereby diminishing the rate of unanticipated postoperative events. However, it seems that surgical decompression alone can expedite the clinical recovery of this subset of patients compared to decompression with instrumentation. 
Furthermore, it is important to highlight the occurrence of secondary instability after surgical spinal decompression, especially in older patients. Fox et al. suggested that radiologically confirmed secondary instability after spinal decompression is a common phenomenon that correlates with worse clinical outcomes or the necessity of additional fusion surgery [11]. Patients with preoperative instability signs, such as preoperative anterolisthesis or abnormal motion, on dynamic radiography, are at a higher risk of secondary instability after a spinal decompression procedure [16]. In our study, the mean follow-up was $>2$ years, and no additional surgeries due to secondary instability were performed. We believe that advances in surgical techniques over the past decade, allowing adequate decompression with minimal disruption to the surrounding stabilizing factors, and the exclusion of patients with preoperative instability signs could have been reasons for the lack of revision surgeries in such a frail cohort. Nevertheless, a quite frequent phenomenon observed in older adults is the progressive degeneration of the spine as well as the spontaneous fusion, which might have contributed to higher rates of stability [4].

\section{Strengths and limitations}

The main strength of the current study is that we are the first to examine the outcomes of octogenarians undergoing surgery for SEA. However, this study has some limitations. First, we examined a relatively small cohort of patients. Nevertheless, since there is a lack of robust evidence of the clinical course of such a devastating disease in older individuals, we believe that our findings greatly clarify the clinical picture. Second, the minimum follow-up period of 12 months was relatively short; by gathering long-term data, other relevant findings not captured in the current study might have been revealed. Third, as this is a retrospective study, selection bias may have been present. Larger studies might be needed to elucidate potential candidates for non-operative management with antibiotic therapy only.

\section{Implications and future directions}

Based on our observation of a fast recovery in patients undergoing surgical decompression only, one might argue that surgical decompression might be the key treatment strategy for the patients studied here. However, we believe a multidisciplinary approach primarily involving experienced spine surgeons is needed to determine an adequately individualized therapy for frail older patients with severe baseline histories. Larger studies are needed to investigate the trends observed in our patient population further.

\section{Conclusions}

Due to a steadily increasing average life expectancy, spine surgeons frequently encounter older patients requiring surgical therapy. Our results show that surgical decompression only and decompression with instrumentation can be considered safe treatments strategies for patients with SEA older than 80 years. It seems that older patients undergoing only surgical decompression might recover neurologically and clinically more quickly than patients who had additional instrumentation. However, the surgical treatment should be clearly discussed with the patient and their relatives. We hope this study will serve as a basis for developing a consensus on the best treatment strategy for frail patients confronting such a devastating illness.

\section{Declarations}


Conflicts of Interest All authors declare that they have no conflicts of interest.

Funding There was no external funding for the presented work

Ethics Approval This study was conducted in accordance with the Declaration of Helsinki and approved by the local ethics committee (S-880/2021).

Consent to participate The requirement for informed consent was waived because of the retrospective nature of this study.

Consent to participate No individual person's data were included in this study

Data Material Availability The datasets generated during and/or analyzed during the current study are available from the corresponding author on reasonable request.

Authors' contributions All authors contributed to the study conception and design. Material preparation, data collection and analysis were performed by Basem Ishak and Pavlina Lenga. The first draft of the manuscript was written by Pavlina Lenga and $\mathrm{PL}, \mathrm{GG}, \mathrm{ABK}, \mathrm{MI}, \mathrm{RO}, \mathrm{JC}, \mathrm{KK}, \mathrm{AU}, \mathrm{BI}$ commented on previous versions of the manuscript. All authors read and approved the final manuscript.

\section{References}

1. Alton TB, Patel AR, Bransford RJ, Bellabarba C, Lee MJ, Chapman JR (2015) Is there a difference in neurologic outcome in medical versus early operative management of cervical epidural abscesses? Spine J 15:10-17. doi: 10.1016/j.spinee.2014.06.010

2. Arko L, Quach E, Nguyen V, Chang D, Sukul V, Kim B-S (2014) Medical and surgical management of spinal epidural abscess: a systematic review. Neurosurg Focus 37:E4. doi: 10.3171/2014.6.FOCUS14127

3. Baker AS, Ojemann RG, Swartz MN, Richardson EP (1975) Spinal epidural abscess. N Engl J Med 293:463-468. doi: 10.1056/NEJM197509042931001

4. Benoist M (2003) Natural history of the aging spine. Eur Spine J 12:S86-S89. doi: 10.1007/s00586-0030593-0

5. Boody BS, Jenkins TJ, Maslak J, Hsu WK, Patel AA (2015) Vertebral Osteomyelitis and Spinal Epidural Abscess: An Evidence-based Review. J Spinal Disord Tech 28:E316-327. doi:

10.1097/BSD.0000000000000294

6. Connor DE, Chittiboina P, Caldito G, Nanda A (2013) Comparison of operative and nonoperative management of spinal epidural abscess: a retrospective review of clinical and laboratory predictors of neurological outcome. J Neurosurg Spine 19:119-127. doi: 10.3171/2013.3.SPINE12762

7. Darouiche RO (2006) Spinal epidural abscess. N Engl J Med 355:2012-2020. doi: 10.1056/NEJMra055111

8. Davis DP, Wold RM, Patel RJ, Tran AJ, Tokhi RN, Chan TC, Vilke GM (2004) The clinical presentation and impact of diagnostic delays on emergency department patients with spinal epidural abscess. J Emerg Med 26:285-291. doi: 10.1016/j.jemermed.2003.11.013

Page 12/14 
9. Deyo RA, Cherkin DC, Ciol MA (1992) Adapting a clinical comorbidity index for use with ICD-9-CM administrative databases. J Clin Epidemiol 45:613-619. doi: 10.1016/0895-4356(92)90133-8

10. Du JY, Schell AJ, Kim C-Y, Trivedi NN, Ahn UM, Ahn NU (2019) 30-day Mortality Following Surgery for Spinal Epidural Abscess: Incidence, Risk Factors, Predictive Algorithm, and Associated Complications. Spine (Phila Pa 1976) 44:E500-E509. doi: 10.1097/BRS.0000000000002875

11. Fox MW, Onofrio BM, Onofrio BM, Hanssen AD (1996) Clinical outcomes and radiological instability following decompressive lumbar laminectomy for degenerative spinal stenosis: a comparison of patients undergoing concomitant arthrodesis versus decompression alone. J Neurosurg 85:793-802. doi: 10.3171/jns.1996.85.5.0793

12. de Groot V, Beckerman H, Lankhorst GJ, Bouter LM (2003) How to measure comorbidity. a critical review of available methods. J Clin Epidemiol 56:221-229. doi: 10.1016/s0895-4356(02)00585-1

13. Heusner AP (1948) Nontuberculous spinal epidural infections. N Engl J Med 239:845-854. doi: 10.1056/NEJM194812022392301

14. Ishak B, Younsi A, Wieckhusen C, Slonczewski P, Unterberg AW, Kiening KL (2019) Accuracy and revision rate of intraoperative computed tomography point-to-point navigation for lateral mass and pedicle screw placement: 11-year single-center experience in 1054 patients. Neurosurg Rev 42:895-905. doi: 10.1007/s10143-018-01067-z

15. Kim JH, Lee EH, Kim J, Kim C (2020) Clinical Outcome of Spinal Epidural Abscess in Elderly Patients. Nerve 6:42-49. doi: 10.21129/nerve.2020.6.2.42

16. Mosenthal WP, Dickherber JL, Saitta BH, Lee MJ (2019) Post laminectomy instability. Seminars in Spine Surgery 31:100713. doi: 10.1053/j.semss.2019.04.007

17. Nussbaum ES, Rigamonti D, Standiford H, Numaguchi Y, Wolf AL, Robinson WL (1992) Spinal epidural abscess: a report of 40 cases and review. Surg Neurol 38:225-231. doi: 10.1016/0090-3019(92)90173-k

18. Patel AR, Alton TB, Bransford RJ, Lee MJ, Bellabarba CB, Chapman JR (2014) Spinal epidural abscesses: risk factors, medical versus surgical management, a retrospective review of 128 cases. Spine J 14:326330. doi: 10.1016/j.spinee.2013.10.046

19. Pereira CE, Lynch JC (2005) Spinal epidural abscess: an analysis of 24 cases. Surg Neurol 63(Suppl 1):S26-29. doi: 10.1016/j.surneu.2004.09.021

20. Reihsaus E, Waldbaur H, Seeling W (2000) Spinal epidural abscess: a meta-analysis of 915 patients. Neurosurg Rev 23:175-204 discussion 205. doi: 10.1007/pl00011954

21. Rigamonti D, Liem L, Sampath P, Knoller N, Namaguchi Y, Schreibman DL, Sloan MA, Wolf A, Zeidman S (1999) Spinal epidural abscess: contemporary trends in etiology, evaluation, and management. Surg Neurol 52:189-196 discussion 197. doi: 10.1016/s0090-3019(99)00055-5

22. Roßbach BP, Niethammer TR, Paulus AC, Melcher C, Birkenmaier C, Jansson V, Wegener B (2014) Surgical treatment of patients with spondylodiscitis and neurological deficits caused by spinal epidural abscess (SEA) is a predictor of clinical outcome. J Spinal Disord Tech 27:395-400. doi: 10.1097/BSD.0000000000000030

23. Vakili M, Crum-Cianflone NF (2017) Spinal Epidural Abscess: A Series of 101 Cases. Am J Med 130:1458-1463. doi: 10.1016/j.amjmed.2017.07.017 


\section{Figures}

\section{Figure 1}

Postcontrast sagittal (A) and axial (B) magnetic resonance (T1 gadolinium sequence) imaging of dorsal lumbar epidural abscess and early end plate destruction of $L 2$ and $L 3$ of an 85-year-old male patient presenting with lumbar pain and progressive low extremety weakness. 